IJOT

46,1

4

Received 3 July 2017

Revised 3 October 2017

17 November 2017

Accepted 20 November 2017

\section{Association between older adults' functional performance and their scores on the Mini Mental State Examination (MMSE) and Montreal Cognitive Assessment (MoCA)}

Sze Tim Sonia Yu, Mong-lin Yu and Ted Brown

Department of Occupational Therapy, School of Primary and Allied Health Care, Faculty of Medicine, Nursing and Health Sciences, Monash University, Victoria, Australia, and

Hanna Andrews

Casey Hospital, Monash Health, Berwick, Victoria, Australia

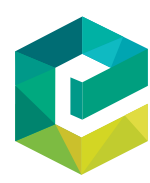

Irish Journal of Occupational Therapy Vol. 46 No. 1, 2018

pp. 4-23
Emerald Publishing Limited Emerald Put DOI 10.1108/IJOT-07-2017-0020

\begin{abstract}
Purpose - The paper aims to investigate if the performance of older adults on the Mini-Mental State Examination (MMSE) and the Montreal Cognitive Assessment (MoCA) were associated or predictive of their functional performance in a geriatric evaluation and management (GEM) inpatient hospital setting. This will inform the occupational therapy assessment and management of older adults admitted to sub-acute GEM settings.

Design/methodology/approach - In all, 20 participants (11 men, 9 women, mean age 82 years, SD = 6.93) were recruited from a GEM ward in an Australian hospital. Participants' cognitive abilities were assessed using the MMSE and MoCA, and their functional performance were assessed using the Functional Independence Measure (FIM). Spearman's rho correlations and linear regression analyses were completed. Bootstrapping was applied to the regression analyses to accommodate the small study sample size.
\end{abstract}

Findings - No statistically significant correlations were obtained between the total and subscale scores of the MMSE and FIM or between the total and subscale scores of the MoCA and FIM. In other words, the cognitive and functional abilities of older adults admitted to a GEM setting were not significantly associated in this study.

Originality/value - The findings suggest that the MoCA and the MMSE were not predictive of participants' functional performance as measure by the FIM in a sub-acute GEM setting. Occupational therapists should be cautious when interpreting participants' MMSE, MoCA and FIM results and not depend

(c) Sze Tim Sonia Yu, Mong-Lin Yu, Ted Brown and Hanna Andrews. Published in Irish Journal of Occupational Therapy. Published by Emerald Publishing Limited. This article is published under the Creative Commons Attribution (CC BY 4.0) licence. Anyone may reproduce, distribute, translate and create derivative works of this article (for both commercial and non-commercial purposes), subject to full attribution to the original publication and authors. The full terms of this licence may be seen at $\mathrm{http} / / /$ creativecommons.org/licences/by/4.0/legalcode

The authors would like to sincerely acknowledge all participants and their families who volunteered to take part in this study. 
solely on these results in the goal setting and intervention planning processes for clients on GEM wards. Further studies are recommended to confirm these findings.

Keywords Assessment, Cognition, Older adults, Occupational therapy, Function, Occupational performance

Paper type Research paper

\section{Introduction}

Geriatric evaluation and management (GEM) is a type of sub-acute care that focuses mainly on patients' functional abilities and goals and caters for patients who have prolonged or multiple conditions associated with ageing, cognitive impairment, functional decline, chronic illness or disability. Patients admitted to GEM wards commonly present with acute conditions (e.g. pneumonia and fractures of the femur, lumbar spine and pelvis) and chronic conditions (e.g. heart failure and chronic obstructive pulmonary disease) [Australian Institute of Health and Welfare (AIHW), 2013]. Dementia and other forms of cognitive impairment are also common comorbidities for patients admitted to sub-acute facilities (Bloomer and Digby, 2012). Often the cognitive skills and functional performance of older adults admitted to GEM settings are assessed. Therefore, the aim of this study was to investigate if the performance of older adults admitted to an inpatient GEM hospital unit on the Mini-Mental State Examination (MMSE) and the Montreal Cognitive Assessment (MoCA) were associated or predictive of their functional performance (as measured by the Functional Independence Measure [FIM]).

Cognition refers to the integrated mental processes comprising the acquisition and use of knowledge, which brings about thoughts and goal directed actions. It is directly related to one's ability to engage in activities of daily life and participate in purposeful, proactive interactions and complex decision making (Radomski and Morrison, 2014). This close relationship between one's cognition and performance and participation in daily activities is also exhibited in a number of practice models and frameworks of occupational therapy, which provide a systematic and comprehensive way to conceptualize and guide occupational therapy practice. The Occupational Therapy Practice Framework, third edition (OTPF-III; American Occupational Therapy Association [AOTA], 2014), and the Canadian Model of Occupational Performance and Engagement (CMOP-E; Townsend and Polatajko, 2013) are examples that highlighted that cognitive abilities can have powerful effects on one's daily occupational performance and participation, in addition to physical skills, environments, occupations or daily activities and other relevant factors.

Several studies also discovered that individuals with cognitive impairment have more difficulties when participating in everyday activities than those individuals without cognitive impairment. Wadley et al. (2008) conducted a comparison group study of older adults with and without mild cognitive impairment on the speed and accuracy in performing some common instrumental activities of daily living (IADLs) which included telephone use, locating nutrition information on food labels, financial abilities, grocery shopping and medication management. The study revealed that individuals with cognitive impairment demonstrated accuracy comparable with cognitively normal individuals but required much more time to complete the IADLs, as these activities depend heavily on memory and complex reasoning.

Likewise, Aretouli and Brandt's (2010) study which compared the functional abilities between 124 elders with cognitive impairment and 68 cognitively normal elders found out that more than one third of the participants with cognitive impairment had difficulties in keeping appointments, finding their belongings, remembering current events and following
Mini Mental State Examination 
IJOT

46,1

television programs. Other reported difficulties include driving and using public transportation, managing finances responsibly and planning and sequencing activities (Aretouli and Brandt, 2010). In severe cases, individuals with cognitive impairment may even have difficulties in basic activities of daily living, such as eating, toileting, showering and dressing [Australian Institute of Health and Welfare (AIHW), 2015]. As a result, these functional limitations may influence the independence, safety and quality of life of the individuals and increase the burden of their careers and the health and social care systems (Gauthier et al., 2006; Reppermund et al., 2013).

For these reasons, occupational therapists play an important role in sub-acute and GEM settings, as they are one of the health professionals that are responsible for identifying signs of early dementia and/or cognitive impairment and providing relevant feedback to the multidisciplinary team, so as to facilitate appropriate, informed and safe discharge planning for the patients (Douglas et al., 2007). To detect cognitive impairment, cognitive assessments are often used, as they can also assist occupational therapists in determining an individual's baseline cognitive status, monitoring disease progression and identifying the individual's cognitive strengths and weaknesses affecting he/she occupational performance (Radomski and Morrison, 2014).

Some literature exploring the relationship between cognitive assessment results and functional performance has been published previously. Toglia et al.'s (2011) study were the first study to explore the relationships of the MMSE and MoCA with functional performance in persons with stroke. This study included 72 inpatients with stroke and mild neurologic and cognitive deficits recruited from an acute rehabilitation unit of a hospital in the USA. It revealed that the MoCA had a higher sensitivity, less of a ceiling effect, higher internal reliability and marginally stronger associations with discharge functional status than the MMSE.

Likewise, a quantitative study of 30 participants recruited from three acute care hospitals in Australia explored the association between the MMSE and the functional performance of inpatients with suspected dementia and suggested that the MMSE scores of inpatients with suspected dementia were significantly associated with their functional performance (Brown et al., 2014b). However, the study noted that therapists need to be cautious when using the MMSE scores to predict the motor task performance of a patient. Also, the results of this study might not be generalized to the population with suspected dementia due to its small sample size and the uneven distribution of male and female participants (Brown et al., 2014b). Therefore, it is important to further investigate whether the MMSE and MoCA are predictive of clients' functional performance and comparing the predictability of both assessments as suggested by previous studies (Brown et al., 2014b; Toglia et al., 2011). The MoCA and the MMSE are both well-known cognitive assessment scales, and the FIM is one of the most widely used functional outcome measures in health care (Brown et al., 2015; Chumney et al., 2010; Glenny and Stolee, 2009; Meyer et al., 2015; Wales et al., 2016). Establishing whether there is a formal link between clients' cognitive skills and their functional performance will inform the clinical practice of newly graduated occupational therapists.

\section{Method \\ Participants}

In total, 20 participants were recruited from a GEM ward in a hospital in Melbourne, Australia, using a convenience sampling approach. Only patients who were aged 65 years or over were able to read and write and had sufficient English competence required for understanding the questions on the MMSE and MoCA and provided consent to participate 
in the study if they did not have a confirmed diagnosis of dementia or other forms of cognitive impairment or have signed consent from their next of kin/family member if they were suspected of having cognitive impairment were recruited. Patients were excluded if they had a confirmed diagnosis of dementia or other forms of cognitive impairment or a secondary/pre-existing neurological medical condition (e.g. Multiple Sclerosis or Parkinson's disease), were medically/psychiatrically unstable, in delirium, or profoundly deaf or blind, or were not able to complete both the MMSE and MoCA.

\section{Instrumentation}

Mini-Mental State Examination (Folstein et al., 1975). The MMSE is a widely used standardized cognitive screening scale designed to detect cognitive impairment. It has 11 questions that assess the cognitive domains of orientation to time and place, registration, attention/calculation, recall, language (including naming, repetition, comprehension, reading and writing) and copying. The total score is 30, with a score of 24 or below being suggestive of cognitive impairment (Folstein et al., 1975; Radomski and Morrison, 2014). The MMSE is a quick assessment, taking approximately 5-10 min to administer. Additionally, the MMSE was reported to have high internal consistency for older adults with cognitive impairment (Cronbach's alpha $=0.81$ ) (Tombaugh et al., 1996) and for cancer patients (Cronbach's alpha $=0.89)$ (Mystakidou et al., 2007). High 24-h test-retest reliability (Pearson $r=0.88, p<$ 0.05) and adequate to high inter-rater reliability were also reported (Folstein et al., 1975; Molloy and Standish, 1997), together with good concurrent and construct validity (Folstein et al.,1975; McPherson et al., 1997; Razani et al., 2009). However, Tombaugh and McIntyre (1992) indicated that the MMSE has low sensitivity for mild cognitive impairment, and its score may be affected by age, education and cultural background of respondents. The MMSE has also been shown to have a negative bias toward individuals from culturally and linguistically diverse backgrounds (Moraes et al., 2012; Naqvi et al., 2015).

Montreal Cognitive Assessment (Nasreddine et al., 2005). The MoCA is a relatively new cognitive screening tool developed to detect mild cognitive impairment. It measures executive functions, visuospatial skills, language, attention and concentration, calculations, memory and delayed recall, conceptual thinking and orientation. The maximum score is 30, with a score of 26 or below indicative of cognitive impaired (Nasreddine et al., 2005; Radomski and Morrison, 2014). The MoCA takes 10-15 min to administer and has good psychometric properties. Nasreddine et al. (2005), and Gill et al. (2008) indicated that the MoCA has excellent test-retest reliability, internal consistency and inter-rater reliability. The MoCA is also evidenced to have excellent concurrent validity, discriminant validity, adequate predictive validity of functional status, as well as high sensitivity in identifying mild cognitive impairment (Gill et al., 2008; Nasreddine et al., 2005; Radomski and Morrison, 2014; Toglia et al., 2011). The MoCA has been translated into 36 different languages and has been used in many different cross cultural settings (Julayanont et al., 2012; Nasreddine et al., 2005; Zheng et al., 2012).

Functional Independence Measure (Granger et al., 1993). The FIM is a widely used outcome measure to determine the degree of disability that individuals experience when participating in functional tasks (Granger et al., 1993; University of Wollongong, 2014). It includes 18 items in total and these items are grouped into two themes: motor and cognitive. The motor group contains 13 items in the areas of self-care (eating, grooming, bathing, dressing upper body, dressing lower body and toileting), sphincter control (bladder management and bowel management), transfers (bed and chair transfers, toilet transfers and tub/shower transfers) and locomotion (walk/wheelchair and stairs) (Granger et al., 1993). The cognitive group comprises five items in the areas of communication (comprehension
Mini Mental State Examination 
IJOT

46,1

and expression) and social cognition (social interaction, problem-solving and memory) (Granger et al., 1993).

Each item is assessed against a seven-point ordinal scale with a score of 1 representing total assistance and a score of 7 representing complete independence. The total score ranges from 18 to 126 , and the scores are based on clinical observation (Granger et al., 1993). The FIM can be utilized within a multidisciplinary team, and it takes approximately $45 \mathrm{~min}$ to complete. The FIM was reported to have excellent test-retest reliability (intra-class correlation coefficient = 0.98) (Hobart et al., 2001), excellent inter-rater reliability (median correlations coefficients $=0.95$ ) (Ottenbacher et al., 1996) and high internal consistency (Cronbach's alpha $=0.88$ admission; 0.91 discharge) (Hsueh et al., 2002). A strong correlation between the FIM motor subscale, and the ten-item version of the Barthel Index (BI) (Spearman's correlation coefficient $=0.92$ ) were also reported indicating high concurrent validity (Hsueh et al., 2002). Good construct validity was also reported for the FIM (Stineman et al., 1996).

\section{Data entry, management and analysis}

The Statistical Package for the Social Sciences version 20 for Windows (IBM Corp, 2011) was used for data entry, storage and analysis. Descriptive statistics, Spearman's rho correlation and linear regression analyses were completed to investigate the association between participants' MMSE and MoCA scores and their FIM scores. Statistical significance was set at $p<0.05$.

To complete a linear regression analysis, the sample size plays an important role, as it affects the generalizability of the regression findings (Pallant, 2016). Therefore, to validate the use of linear regression models for purposes of prediction in this study, a resampling technique referred to as bootstrapping was used. Bootstrapping is a type of robust statistic that infers a population from sample data (Hinton et al., 2014). It works by taking, with replacement, the values from the original sample to obtain thousands of bootstrapped samples to improve the accuracy of the confidence interval $(\mathrm{CI})$ estimation for one or more statistics (Field, 2013; Hinton et al., 2014). When performing bootstrapping, it is assumed that the original sample reasonably represents the population (LaFlair et al., 2015).

In this study, although a convenient sampling approach was used, the participants recruited were equally distributed males and females, and from a range of cultural backgrounds which are at least partially representative of the cultural and linguistic diversity of the Australian population. According to LaFlair et al. (2015), bootstrapping can be applied to a variety of statistical tests, including regression analysis, and it is a powerful non-parametric analytical tool when researchers are faced with limitations of small sample sizes. Thus, all regression analyses performed in this study used the technique of bootstrapping and were based on bootstrapped sample size of 1,000 to minimize the limitation of a small sample size.

\section{Procedures}

Ethical approval was obtained through both Monash Health and Monash University Human Research Ethics committees. All ethical issues relevant to this study were carefully considered and minimized by applying corresponding strategies. Participants who met the specified inclusion criteria were identified by their treating occupational therapists. Consent was obtained from all participants and their next of kin if the participants were deemed as suspected of having cognitive impairment by their treating occupational therapists.

Participant's demographic data, including age, gender, country of birth, language spoken at home and reason(s) for admission, were collected via their medical file. To ensure the reliability and accuracy of the data and minimize inconsistent scoring, the first author 
completed the two cognitive assessments (the MMSE and MoCA) with all participants. The FIM was completed by the participant's treating multi-disciplinary team as trainings are required to administer the FIM. As the FIM was only completed upon the participant's admission and prior to discharge from the sub-acute ward, the two cognitive assessments were completed within a week after the FIM was completed either at admission or prior to discharge to ensure the participant's medical condition did not have much variation which then affected the accuracy of the cognitive assessment results. All assessments were completed in accordance with the guidelines and protocols outlined in the MMSE, MoCA and FIM manuals.

\section{Results}

\section{Participants}

A total of 20 participants $(N=20)$ took part in the study. Of the participants, 11 were male (55 per cent) and 9 were female ( 45 per cent). The age of the participants ranged from 66 to 93 years, with a mean age of 82.05 years (standard deviation $[S D]=6.93$ ). All participants spoke English with the majority of them being born in Australia $(n=10,50$ per cent) or the UK $(n=5,25$ per cent). The most common reason for the participants' admission to hospital was orthopaedic conditions secondary to a fall ( $n=11,55$ per cent), followed by general deconditioning ( $n=2,10$ per cent), cardiac conditions ( $n=1,5$ per cent) and other medical issues $(n=6,30$ per cent).

\section{Participant instrument scores}

The mean, median, SD, range and interquartile ranges of the scores from the MMSE, MoCA and FIM are detailed in Table I. The mean scores for the MMSE scales were as follows: orientation 9.35 (SD 0.75), registration 3.0 (SD 0.0), attention/calculation 2.90 (SD 1.89), delayed recall 2.40 (SD 0.68), language 7.30 (SD 0.86), copying 0.90 (SD 0.31) and total 25.85 (SD 2.68), whereas for the MoCA, they were executive functions and visuospatial skills 3.50 (SD 1.10), naming 2.60 (SD 0.60), attention 4.75 (SD 1.37), language 1.75 (SD 0.72), abstraction 1.10 (SD 0.72), delayed recall 1.15 (SD 1.39), orientation 5.65 (SD 0.59) and total 20.50 (SD 3.19). For the FIM, the mean scores for the total, motor and cognitive scales were 53.0 (SD 13.09), 31.3 (SD 4.29) and 84.3 (SD 15.96), respectively.

\section{Correlation results}

The associations of the total scores and subscale scores of the MMSE and MoCA to the total and subscale scores of the FIM were investigated using the Spearman's rho correlation coefficients. Tables II provide details of the Spearman's rho correlation coefficients results. The correlation analyses indicated that there were no statistically significant correlations identified between the MMSE and MoCA total scores and subscale scores and the FIM total and subscale scores. The non-significant Spearman rho correlations between the MMSE and FIM scales ranged between -0.13 to 0.35 , whereas between the MoCA and FIM scales the non-significant coefficients ranged from -0.22 to 0.25 .

\section{Regression results}

Although there were no statistically significant correlations identified between the MMSE and FIM, and between the MoCA and FIM, multiple linear regression analyses using the bootstrapping technique were still carried out utilizing the original study participant sample $(N=20)$ to further investigate if the MMSE and MoCA subscale scores were predictive of participants' functional performance. A series of regression analyses were competed using
Mini Mental State Examination 


\section{IJOT \\ 46,1}

\begin{tabular}{|c|c|c|c|c|c|}
\hline Instrument subscale and total scores & Mean & Median & $\mathrm{SD}$ & Range & $\begin{array}{l}\text { Interquartile range } \\
\qquad\left(\mathrm{Q}_{1}, \mathrm{Q}_{3}\right)\end{array}$ \\
\hline \multicolumn{6}{|l|}{ MMSE } \\
\hline Orientation & 9.35 & 9.00 & 0.75 & $7-10$ & 9,10 \\
\hline Registration & 3.00 & 3.00 & 0.00 & $3-3$ & 3,3 \\
\hline Attention/Calculation & 2.90 & 2.50 & 1.89 & $0-5$ & 1,5 \\
\hline Delayed recall & 2.40 & 2.50 & 0.68 & $1-3$ & 2,3 \\
\hline Language & 7.30 & 8.00 & 0.86 & $6-8$ & $6.25,8$ \\
\hline Copying & 0.90 & 1.00 & 0.31 & $0-1$ & 1,1 \\
\hline Total & 25.85 & 25.50 & 2.68 & $21-30$ & 24,28 \\
\hline \multicolumn{6}{|l|}{$\mathrm{MoCA}$} \\
\hline Executive functions and visuospatial skills & 3.50 & 3.00 & 1.10 & $2-5$ & $3,4.75$ \\
\hline Naming & 2.60 & 3.00 & 0.60 & $1-3$ & 2,3 \\
\hline Attention & 4.75 & 5.00 & 1.37 & $1-6$ & 4,6 \\
\hline Language & 1.75 & 2.00 & 0.72 & $1-3$ & 1,2 \\
\hline Abstraction & 1.10 & 1.00 & 0.72 & $0-2$ & 1,2 \\
\hline Delayed recall & 1.15 & 0.50 & 1.39 & $0-4$ & $0,2.75$ \\
\hline Orientation & 5.65 & 6.00 & 0.59 & $4-6$ & 5,6 \\
\hline Total & 20.50 & 21.00 & 3.19 & $13-25$ & 19,23 \\
\hline \multicolumn{6}{|l|}{ FIM } \\
\hline Motor & 53.00 & 50.00 & 13.09 & $34-80$ & $44.25,59.75$ \\
\hline Cognitive & 31.30 & 33.00 & 4.29 & $22-35$ & $27.75,34.75$ \\
\hline Total & 84.30 & 82.50 & 15.96 & $57-115$ & $74.25,94.75$ \\
\hline
\end{tabular}

\section{Table I.}

Participant sample mean, median, standard deviation, range, and interquartile range scores of the MMSE and $\operatorname{MoCA}(N=20)$
Notes: MMSE $=$ Mini-Mental State Examination; MoCA $=$ Montreal Cognitive Assessment; FIM = Functional Independence Measure. All values rounded to two decimal places

the MMSE subscale scores and MoCA subscale scores as models to predict the FIM total score, FIM Motor subscale score or FIM Cognitive subscale score. Preliminary analyses were conducted to ensure no violations of the assumptions of normality, linearity, multicollinearity and homoscedasticity occurred.

From the results of the regression analyses between the MMSE and FIM, the MMSE subscale scores and the FIM total score were found not to share a predictive relationship, adjusted $R^{2}=-0.09, F(5,14)=0.69, p=$ not statistically significant $(n s)$. Similarly, the regression results indicated that there were no statistically significant, predictive relationships between the MMSE subscale scores and the FIM Motor score, adjusted $R^{2}=$ $-0.13, F(5,14)=0.57, p=n s$; and the MMSE subscale scores and the FIM Cognitive score, adjusted $R^{2}=-0.01, F(5,14)=0.96, p=n s$. Further details of these regressions are presented in Tables III-V.

From the results of the regression analyses between the MoCA and FIM, no statistically significant regression results were obtained between the MoCA subscale scores and the FIM total score adjusted $R^{2}=-0.31, F(7,12)=0.36, p=n s$. Likewise, the MoCA subscale scores were found not to be able to significantly predict the FIM Motor score, adjusted $R^{2}=-0.37$, $F(7,12)=0.27, p=n s$; and the FIM Cognitive score, adjusted $R^{2}=-0.22, F(7,12)=0.51$, $p=n s$. Further details of these regressions are presented in Tables VI-VIII.

\section{Discussion}

This study aimed to explore if the MMSE or MoCA was correlated or predictive of patients' functional performance as measured by the FIM in a sub-acute inpatient setting. The 


\begin{tabular}{|c|c|c|c|c|}
\hline Instrument & Motor subscale & $\begin{array}{l}\text { FIM } \\
\text { Cognitive subscale }\end{array}$ & Total & State \\
\hline \multicolumn{5}{|l|}{ MMSE } \\
\hline Orientation & 0.11 & 0.35 & 0.19 & \\
\hline Attention/Calculation & 0.03 & -0.11 & 0.05 & \\
\hline Delayed recall & -0.16 & 0.12 & -0.13 & \\
\hline Language & 0.15 & 0.10 & 0.12 & 11 \\
\hline \multirow{2}{*}{$\begin{array}{l}\text { Copying } \\
\text { Total }\end{array}$} & -0.12 & 0.18 & -0.15 & \\
\hline & 0.13 & 0.15 & 0.14 & \\
\hline \multicolumn{5}{|l|}{$\mathrm{MoCA}$} \\
\hline Executive functions and visuospatial skills & 0.13 & 0.07 & 0.12 & \\
\hline Naming & -0.15 & -0.22 & -0.11 & \\
\hline Attention & -0.16 & -0.14 & -0.11 & Table II. \\
\hline Language & 0.06 & 0.00 & 0.04 & Spearman's rho \\
\hline Abstraction & 0.09 & -0.05 & 0.01 & correlation \\
\hline Delayed recall & -0.01 & 0.25 & 0.01 & coefficients (two- \\
\hline Orientation & 0.25 & 0.33 & 0.32 & tailed) between the \\
\hline Total & 0.04 & 0.04 & 0.07 & MMSE/MoCA and \\
\hline \multicolumn{4}{|c|}{$\begin{array}{l}\text { Notes: MMSE = Mini-Mental State Examination; MoCA = Montreal Cognitive Assessment; FIM = } \\
\text { Functional Independence Measure. All correlation coefficients rounded to two decimal places. The MMSE } \\
\text { Registration subscale score was a constant and therefore was deleted from the analysis }\end{array}$} & $\begin{array}{r}\text { FIM total and } \\
\text { subscale scores } \\
(N=20)\end{array}$ \\
\hline
\end{tabular}

findings of the Spearman's rho correlation and regression analyses indicated that no statistically significant relationships were obtained between the total and subscale scores of the MMSE and FIM and between the total and subscale scores of the MoCA and FIM. Hence, neither the MMSE nor the MoCA was significantly correlated to or predictive of older adults' functional performance (as measured by FIM).

These results were surprising, as based on the OTPF-III (AOTA, 2014) and the CMOP-E (Townsend and Polatajko, 2013), cognitive abilities are closely linked to an individual's functional performance and participation in daily activities. Furthermore, Millán-Calenti et al. (2012), who scrutinized the role of cognitive decline as a predictor of functional dependence in a sample of 600 community-dwelling Spanish participants aged 65 or older, reported that the cognitive status of the participants as measured by the MMSE, significantly influenced their functional dependence on basic (such as bathing and toileting) and instrumental (such as using the telephone, taking medications and handling finances) activities of daily living. A lower MMSE score implied a higher loss of ability and a larger impact on carrying out daily activities (Millán-Calenti et al., 2012). Thus, reflecting on this previously reported study, it was initially anticipated that the MMSE scores would correlate and be predictive of participants' functional performance (as measured by their FIM total, motor subscale and cognitive subscale scores). Unexpectedly, the findings of the current study contrasted those of previously reported investigations that correlated similar functional traits of older adults.

However, the current study's findings do concur with results reported by Brown et al. (2014a) who obtained no significant associations between the MMSE scores and the functional performance (as measured by the Modified BI and Activities of Daily Living Questionnaire) in a sample of 28 participants who were suspected of having dementia and were recruited from three acute care hospitals in Australia. Likewise, in another Australian study, Brown et al. (2014b) reported that the MMSE scores of 30 older adults (who were 
IJOT

46,1

12

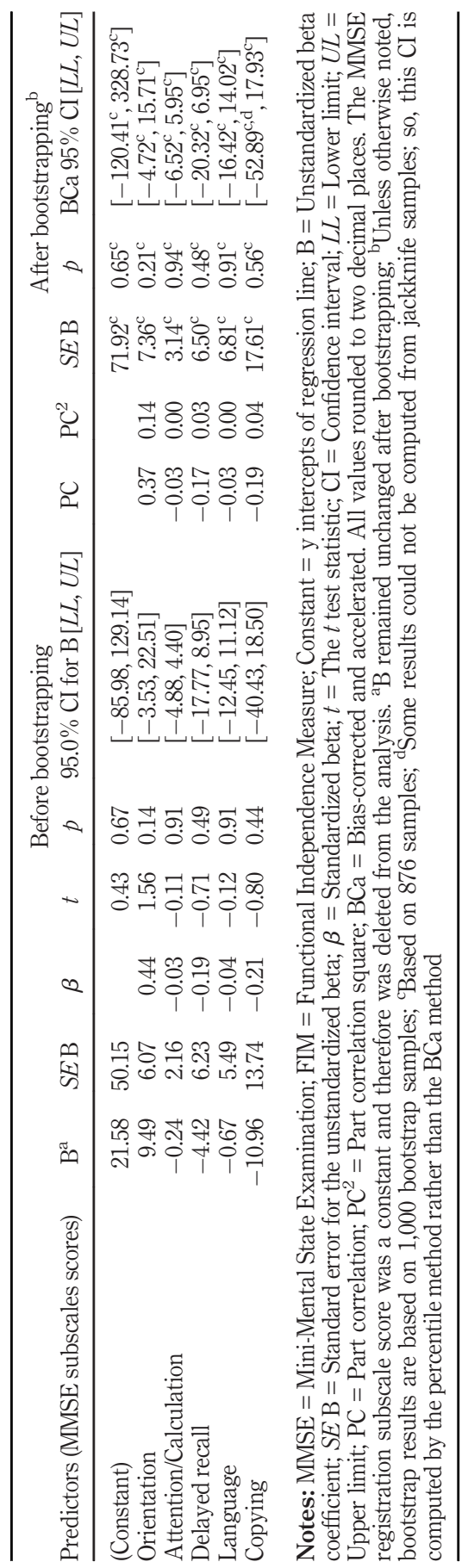

Table III.

Summary of the multiple linear regression analysis between the FIM total score and the MMSE subscale scores $(N=20)$ 


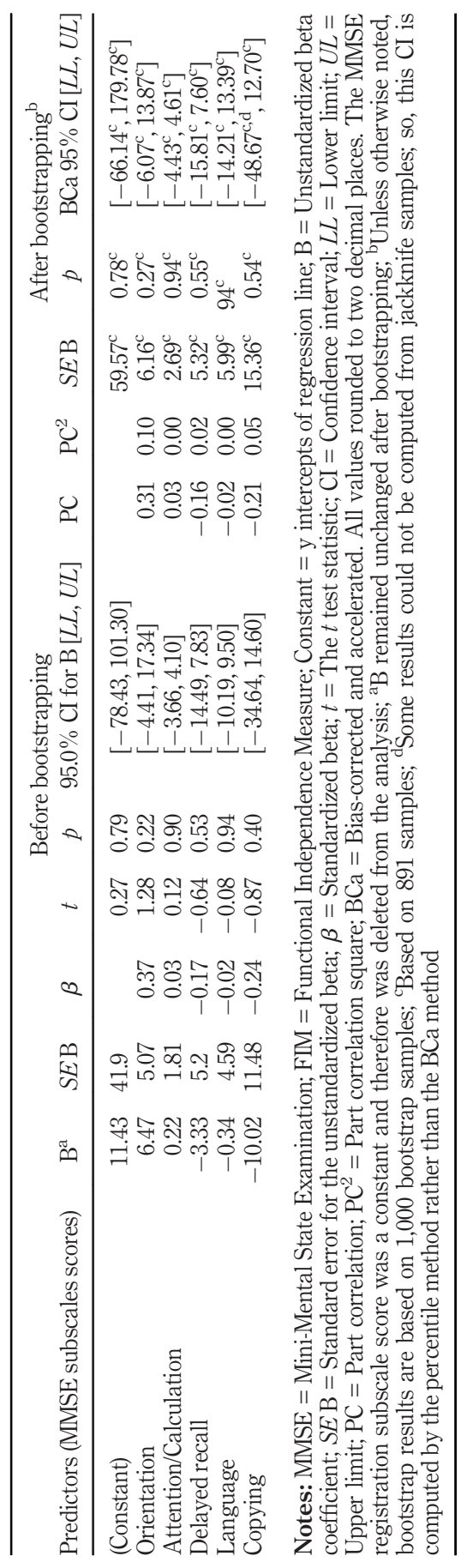

Table IV. Summary of the multiple linear regression analysis between the FIM Motor subscale score and the MMSE subscale scores $(N=20)$ 
IJOT

46,1

14

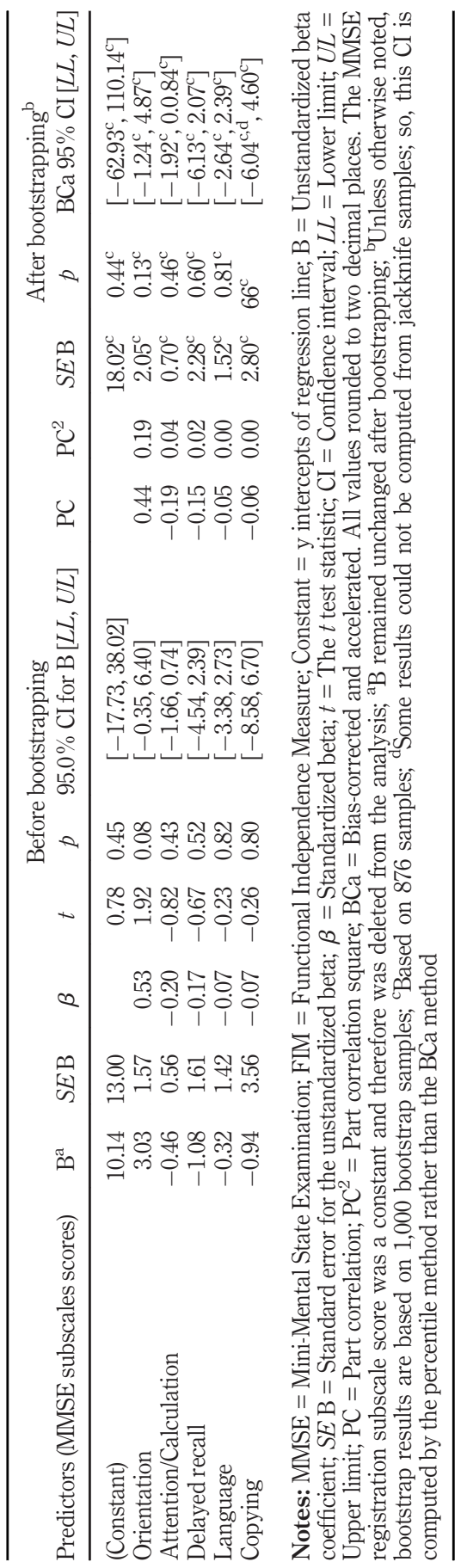

Table V.

Summary of the multiple linear regression analysis between the FIM Cognitive subscale and the MMSE subscale scores $(N=20)$ 


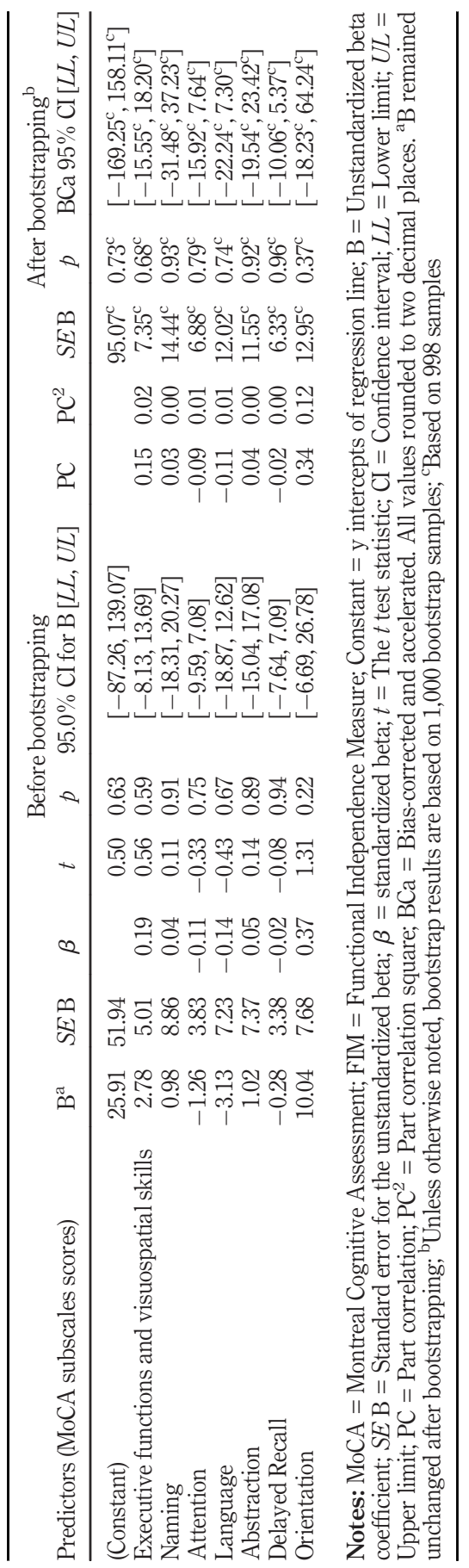

Table VI. Summary of multiple linear regression analysis results between the FIM total score and the MoCA subscale scores $(N=20)$ 
IJOT

46,1

16

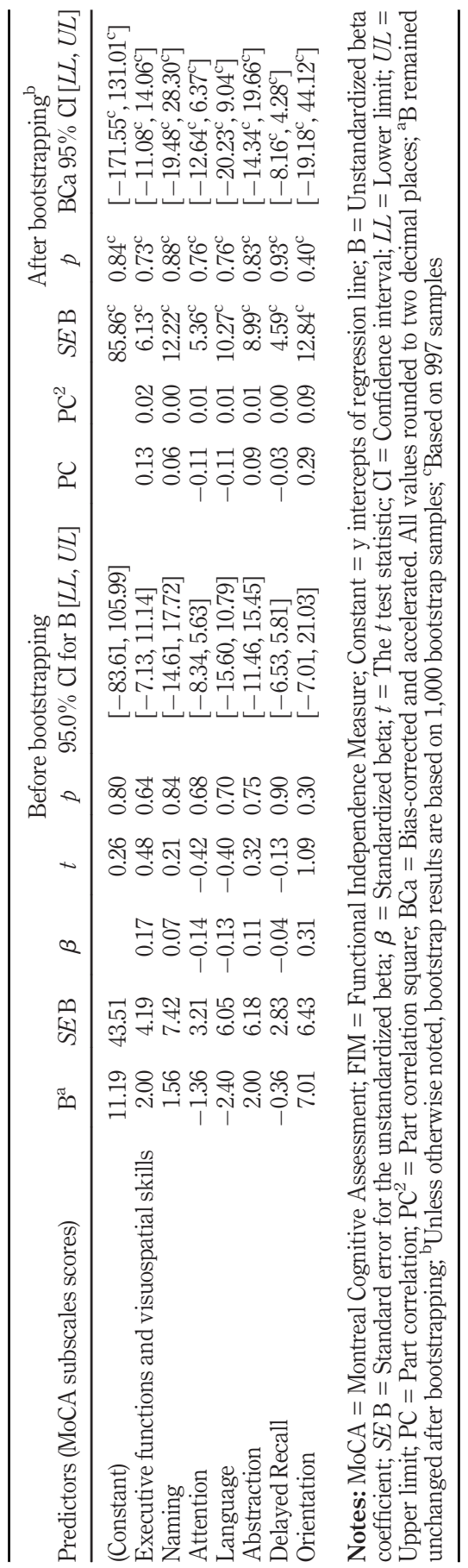

Table VII.

Summary of multiple linear regression analysis between the FIM Motor subscale score and the MoCA subscale scores $(N=20)$ 


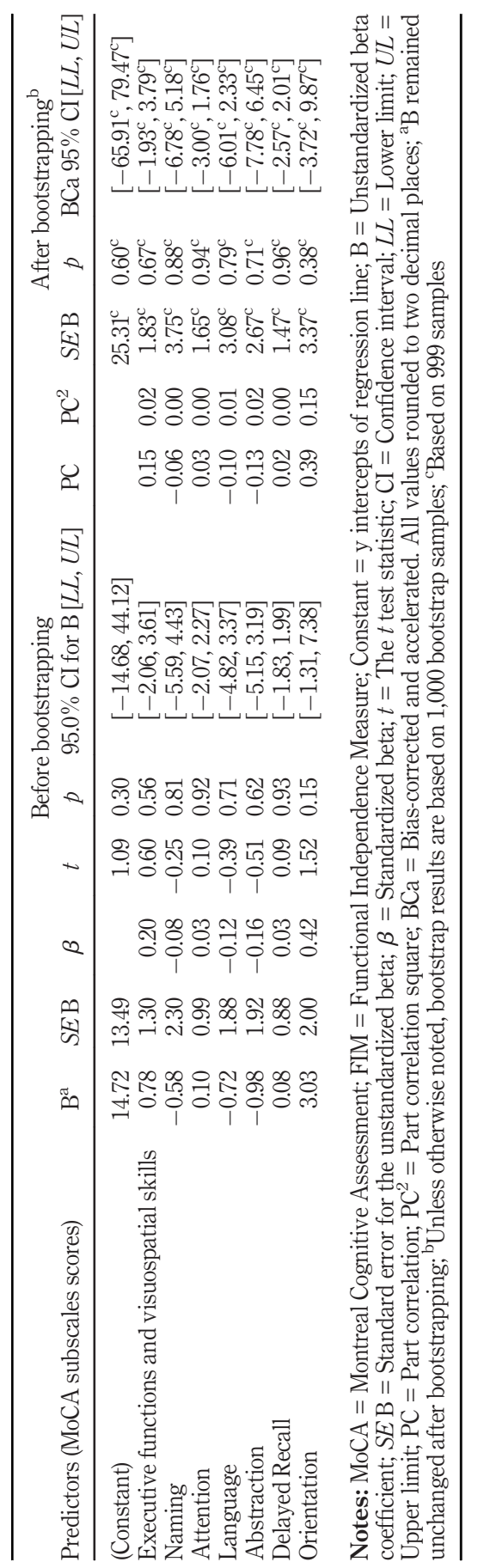

Table VIII. Summary of multiple linear regression analysis between the FIM Cognitive subscale score and the MoCA subscale scores $(N=20)$ 
IJOT

46,1

18

inpatients in acute hospital settings presenting with suspected dementia) were not significantly associated with the FIM Motor subscale score and not predictive of the FIM Motor subscale score. This study indicated that the MMSE may not be a significant predictor of inpatients' motor task performance. The findings of this present study not only support this notion but also demonstrate that the MMSE was not related to the FIM total and Cognitive subscale scores.

However, these findings contrast with some previously published studies. Brown et al. (2014b) did find that the MMSE scores were significantly associated and predictive of the FIM total score $\left(r_{s}=0.37, p<0.05\right.$; adjusted $\left.R^{2}=0.41, p<0.05\right)$ and the FIM Cognitive subscale score $\left(r_{s}=0.61, p<0.01\right.$; adjusted $\left.R^{2}=0.68, p<0.01\right)$. In the same way, Zwecker et al. (2002) and Adunsky et al. (2002) revealed weak to strong significant correlations, $r=$ $0.26(p<0.005)$ and $r=0.57(p<0.001)$, respectively, between the MMSE scores and the FIM Cognitive subscale scores in patients presenting with a stroke. Consequently, these studies indicate that the FIM Cognitive subscale scores can be predicted by the MMSE scores, which is contrary to the findings of this current study. Apart from the FIM Cognitive subscale score, Toglia et al.'s(2011) study also reported that the Spearman's rho correlation coefficient between the MMSE total score and the FIM Motor subscale score was $0.29(p<$ 0.05 ) indicating a weak, but significant association. These findings are different from those of the present study.

The findings of this study compared to others may be explained by the differences of the methodologies, which may have affected the potential for direct comparisons of the study's findings. The majority of the abovementioned studies were conducted with patients diagnosed with a stroke and located in acute hospital settings, whereas this current study was conducted in a sub-acute GEM setting and excluded patients who had pre-existing neurological medical conditions or had been admitted for stroke. Typically, in sub-acute GEM settings, patients often present with prolonged and/or multiple complex health conditions related to ageing (AIHW, 2013). Therefore, the diverse nature of patients' characteristics in acute and sub-acute GEM settings might be one underlying explanation for the diverse findings between the present study and previously reported investigations. As a result, in the context of this study, it is evidence that the MMSE had no statistical connection with the patients' functional performance as measured by the FIM in a sub-acute GEM setting.

Similar to the MMSE, it was postulated that the MoCA scores would correlate and predict the participant's functional performance as indicated in their FIM total, Motor subscale and Cognitive subscale scores. Also, as evidenced in a number of previously reported studies, the $\mathrm{MoCA}$ is considered to have a higher level of sensitivity than the MMSE while retaining specificity in identifying individuals with cognitive impairment and thus is considered to be superior to the MMSE (Sweet et al., 2011; Toglia et al., 2011). Thus, it was expected the MoCA would exhibit a stronger correlation and/or ability to predict the FIM total and subscale scores, as compared to the MMSE. Yet, it was surprising that the present study found no signification relationships between the total and subscales scores of the MoCA and the FIM.

These findings are concordant with parts of the findings established in Sweet et al.'s (2011) study. The MoCA scores were found to have no associations with the FIM Motor subscale score when collected from 47 patients first admitted to the geriatric rehabilitation program in Canada, having a correlation coefficient of $0.04(p>0.05)$. Yet, Sweet et al. (2011) stated that the MoCA scores were significantly correlated to the FIM Cognitive subscale scores when obtained on the participants' admission and upon discharge. The correlation coefficients were $0.37(\phi<0.05)$ and $0.52(\phi<0.05)$, respectively. Also, a moderate level, 
significant correlation was identified between the MoCA scores and the FIM Motor subscale score obtained upon the participants' discharge, with a correlation coefficient of $0.36(p<$ 0.05). Toglia et al. (2011) also obtained similar findings, indicating that the MoCA total score was moderately linked with the FIM Motor subscale score attained on the participant's admission to an acute rehabilitation unit in the USA $\left(r_{s}=0.40, p<0.01\right)$.

The findings of this present study differ from those reported by Sweet et al. (2011) and Toglia et al. (2011). As mentioned above, the participants in Toglia et al.'s(2011) study were patients presenting with a diagnosis of a stroke; so, their clinical presentation was dissimilar to the participants in the current study. Hence, the results reported by Toglia et al. (2011) may not be directly comparable to those of the current study. Besides, Sweet et al.'s (2011) study was the first study to evaluate the ability of the MoCA to predict patients' rehabilitation outcome (as measure by the FIM) in a geriatric rehabilitation setting. Although the participant's characteristics of Sweet et al.'s (2011) study and the current study were similar, Sweet et al. (2011) indicated the need for further research and evaluation of the relationships between the MoCA and the FIM. Even though the findings of the present study found no significant associations between the MoCA and the FIM, further research is needed to confirm the findings.

\section{Implications for practice}

This study indicated that the ability of the MMSE and MoCA in predicting a patient's functional performance (as measured by the FIM) was limited at best; occupational therapists should pay careful attention when interpreting patients' MMSE, MoCA and FIM results together and should not rely solely on these results to assist in the goal setting and intervention planning processes. Even though occupational therapists assumed that one's cognitive status is directly related to one's functional performance in activities of daily life as illustrated in the OTPF-III (AOTA, 2014) and the CMOP-E (Townsend and Polatajko, 2013), patients' cognitive status and functional performance may not be truly reflected by their MMSE, MoCA and FIM results. Other physical comorbidities, psychosocial stressors, unfamiliar environmental cues and other potential related factors need to be taken into account when determining patients' cognitive status and functional performance.

\section{Limitations}

There were several limitations that should be considered when interpreting the findings of this study. First, the sample of this study was small $(N=20)$ and convenience sampling method were used. Moreover, detailed demographic information about the participants, including educational levels, living circumstance and socio-economic status, were not collected. Additionally, the participants of this study were recruited from a sub-acute GEM ward located in a teaching hospital in the metropolitan region of Melbourne, Australia. Therefore, the generalizability of the study's results to the larger population might be affected as only a small geographic region was sampled. However, the participants of this study were from a range of cultural backgrounds which represented the culture and linguistic diversity of the Australian population. As well, the MMSE and MoCA were administered by the researcher which ensured that the cognitive scales were administered in a consistent manner and hopefully minimized the potential for bias occurring.

Another limitation was that the cognitive status of the participants was assessed only at one point in time using the MMSE and MoCA, and thus, their results might not truly reflect their actual cognitive abilities given that their performance might be affected by their illnesses, physical comorbidities, medications, the unfamiliar environment and other potentially related factors. These factors were also applicable when using the FIM to 
IJOT

46,1

measure the participants' functional performance, although comprehensive and extensive exclusion criteria were used to in an attempt to minimize these limitations. Further to this, the participants' functional performance was assessed narrowly by using the FIM, which only consists motor and cognitive items in the areas of self-care, sphincter control, transfers, locomotion, communication and social cognition. Therefore, the areas assessed by the FIM may have not provided a complete overview of the participants' activities of daily living, as these can include personal, domestic and community activities of daily living.

\section{Future research}

Several recommendations for future research are apparent. To increase the generalizability of the result findings, it is recommended to replicate this study with a larger sample size, from a larger geographical region, and utilizing randomized sampling methods. More studies are urged to explore the relationships between the MoCA and the FIM, particularly the individual subscale scores, as well as confirm the finding of this study. In addition, it is recommended to review patients' cognitive status and functional performance at a second time point, so as to allow a comparison of these two sets of factors upon admission and at discharge. This also allows the associations and predictive ability of the MMSE and MoCA and patients' rehabilitation outcomes to be investigated. Furthermore, different or more comprehensive cognitive measures, such as the Addenbrooke's Cognitive Examination-III, and functional assessments, such as the Assessment of Motor and Process Skills, are suggested to be used to evaluate patients' cognitive status and functional performance to allow comparisons between different cognitive assessments and examine if any difference will be made if using a different or more comprehensive cognitive and functional assessments.

\section{Conclusion}

This study revealed that the MMSE and MoCA were found to have no statistically significant associations with patients' functional performance as measured using the FIM. Similarly, the MMSE or MoCA were unlikely to be able to predict patients' functional performance. Thus, occupational therapists should not depend solely on patients' MMSE, MoCA and FIM results to assist in the goal setting and intervention planning processes for patients on GEM wards. Future studies are recommended to further confirm the findings of this study.

\section{References}

Adunsky, A., Fleissig, Y., Levenkrohn, S., Arad, M. and Noy, S. (2002), "Clock Drawing Task, MiniMental State Examination: relation to functional outcome of stroke patients", Archives of Gerontology and Geriatrics, Vol. 35 No. 2, pp. 153-160.

American Occupational Therapy Association (2014), "Occupational Therapy Practice Framework: domain and process (3rd ed.)”, American Journal of Occupational Therapy, Vol. 68 No. 1, pp. S1-S48.

Aretouli, E. and Brandt, J. (2010), "Everyday functioning in mild cognitive impairment and its relationship with executive cognition”, International Journal of Geriatric Psychiatry, Vol. 25 No. 3, pp. 224-233.

Australian Institute of Health and Welfare (AIHW) (2013), "Development of nationally consistent subacute and non-acute admitted patient care data definitions and guidelines", available at: www.aihw.gov.au/WorkArea/DownloadAsset.aspx?id=60129543218 (accessed 1 November 2016). 
Australian Institute of Health and Welfare (AIHW) (2015), "Dementia”, available at: www.aihw.gov.au/ dementia/ (accessed 26 October 2016).

Bloomer, M.J. and Digby, R. (2012), "Caring for patients dying with dementia: challenges in subacute care in Australia", Journal of Palliative Care, Vol. 28 No. 3, pp. 164-168.

Brown, A.W., Therneau, T.M., Schultz, B.A., Niewczyk, P.M. and Granger, C.V. (2015), "Measure of functional independence dominates discharge outcome prediction after inpatient rehabilitation for stroke", Stroke, Vol. 46 No. 4, pp. 1038-1044.

Brown, T., Elliott, S. and Fielding, L. (2014a), "Is there a relationship between Mini-Mental Status

Mini Mental State Examination Examination scores and the activities of daily living abilities of clients presenting with suspected dementia”, Physical \& Occupational Therapy in Geriatrics, Vol. 32 No. 4, pp. 336-352.

Brown, T., Joliffe, L. and Fielding, L. (2014b), "Is the Mini-Mental Status Examination (MMSE) associated with inpatients functional performance?", Physical \& Occupational Therapy in Geriatrics, Vol. 32 No. 3, pp. 228-240.

Chumney, D., Nollinger, K., Shesko, K., Skop, K., Spencer, M. and Newton, R.A. (2010), “Ability of Functional Independence Measure to accurately predict functional outcome of stroke-specific population: systematic review", The Journal of Rehabilitation Research and Development, Vol. 47 No. 1, pp. 17-29.

Douglas, A., Liu, L., Warren, S. and Hopper, T. (2007), "Cognitive assessments for older adults: which ones are used by Canadian therapists and why", Canadian Journal of Occupational Therapy, Vol. 74 No. 5, pp. 370-381.

Field, A.P. (2013), Discovering Statistics Using IBM SPSS Statistics, 4th ed., Sage, London.

Folstein, M.F., Folstein, S.E. and McHugh, P.R. (1975), "Mini-mental state: a practical method for grading the cognitive state of patients for the Clinician", Journal of Psychiatric Research, Vol. 12 No. 3, pp. 189-198.

Gauthier, S., Reisberg, B., Zaudig, M., Petersen, R.C., Ritchie, K., Broich, K., Belleville, S., Brodaty, H., Bennett, D., Chertkow, H., Cummings, J.L., de Leon, M., Feldman, H., Ganguli, M., Hampel, H., Scheltens, P., Tierney, M.C., Whitehouse, P. and Winblad, B. (2006), "Mild cognitive impairment”, Lancet (London, England), Vol. 367 No. 9518, pp. 1262-1270.

Gill, D.J., Freshman, A., Blender, J.A. and Ravina, B. (2008), "The Montreal Cognitive Assessment as a screening tool for cognitive impairment in Parkinson's disease", Movement Disorders, Vol. 23 No. 7, pp. 1043-1046.

Glenny, C. and Stolee, P. (2009), "Comparing the Functional Independence Measure and the interRAI/ MDS for use in the functional assessment of older adults: a review of the literature", $B M C$ Geriatrics, Vol. 9 No. 1, p. 52

Granger, C.V., Hamilton, B.B., Linacre, J.M., Heinemann, A.W. and Wright, B.D. (1993), "Performance profiles of the Functional Independence Measure", American Journal of Physical Medicine \& Rehabilitation, Vol. 72 No. 2, pp. 84-89.

Hinton, P.R., McMurray, I. and Brownlow, C. (2014), SPSS Explained, Routledge, Hove, East Sussex.

Hobart, J.C., Lamping, D.L., Freeman, J., Langdon, D.W., McLellan, D.L., Greenwood, R.J. and Thompson, A.J. (2001), "Evidence-based measurement which disability scale for neurologic rehabilitation?”, Neurology, Vol. 57 No. 4, pp. 639-644.

Hsueh, I.P., Lin, J.H., Jeng, J.S. and Hsieh, C.L. (2002), “Comparison of the psychometric characteristics of the Functional Independence Measure, 5 item Barthel Index, and 10 item Barthel Index in patients with stroke", Journal of Neurology, Neurosurgery \& Psychiatry, Vol. 73 No. 2, pp. 188-190.

IBM Corp (2011), IBM SPSS Statistics for Windows, Version 20.0, IBM Corp, Armonk, NY.

Julayanont, P., Phillips, N., Chertkow, H. and Nasreddine, Z.S. (2012), "The Montreal Cognitive Assessment (MoCA): concept and clinical review”, in Larner, A.J. (Ed.), Cognitive Screening Instruments: A Practical Approach, Springer-Verlag, London, pp. 111-152. 
IJOT

46,1

LaFlair, G.T., Egbert, J. and Plonsky, L. (2015), "A practical guide to bootstrapping descriptive statistics, correlations, $\mathrm{t}$ tests, and ANOVAs", in Plonsky, L. (Ed.), Advancing Quantitative Methods in Second Language Research, Routledge, New York, NY, pp. 46-77.

McPherson, K., Berry, A. and Pentland, B. (1997), "Relationship between cognitive impairments and functional performance after brain injury, as measured by the functional assessment measure (FIM+FAM)", Neuropsychological Rehabilitation, Vol. 7 No. 3, pp. 241-257.

Meyer, M.J., Pereira, S., McClure, A., Teasell, R., Thind, A., Koval, J., Richardson, M. and Speechley, M. (2015), "A systematic review of studies reporting multivariable models to predict functional outcomes after post-stroke inpatient rehabilitation", Disability and Rehabilitation, Vol. 37 No. 15, pp. 1316-1323.

Millán-Calenti, J.C., Tubío, J., Pita-Fernández, S., Rochette, S., Lorenzo, T. and Maseda, A. (2012), "Cognitive impairment as predictor of functional dependence in an elderly sample", Archives of Gerontology and Geriatrics, Vol. 54 No. 1, pp. 197-201.

Molloy, D.W. and Standish, T.I. (1997), “A guide to the standardized Mini-Mental State Examination”, International Psychogeriatrics, Vol. 9 No. S1, pp. 87-94.

Moraes, C., Pinto, J.A., Lopes, M.A., Litvoc, J. and Bottino, C.M. (2012), "Impact of sociodemographic and health variables on Mini-Mental State Examination in a community-based sample of older people", European Archives of Psychiatry and Clinical Neuroscience, Vol. 260 No. 7, pp. 535-542.

Mystakidou, K., Tsilika, E., Parpa, E., Galanos, A. and Vlahos, L. (2007), "Brief cognitive assessment of cancer patients: evaluation of the Mini-Mental State Examination (MMSE) psychometric properties", Psycho-Oncology, Vol. 16 No. 4, pp. 352-357.

Naqvi, R.M., Haider, S., Tomlinson, G. and Alibhai, S. (2015), "Cognitive assessments in multicultural populations using the Rowland Universal Dementia Assessment Scale: a systematic review and meta-analysis", Canadian Medical Association Journal, Vol. 187 No. 5, pp. E169-E175.

Nasreddine, Z.S., Phillips, N.A., Bédirian, V., Charbonneau, S., Whitehead, V., Collin, I., Cummings, J.L. and Chertkow, H. (2005), "The Montreal cognitive assessment, MoCA: a brief screening tool for mild cognitive impairment", Journal of the American Geriatrics Society, Vol. 53 No. 4, pp. 695-699.

Ottenbacher, K.J., Hsu, Y., Granger, C.V. and Fiedler, R.C. (1996), "The reliability of the Functional Independence Measure: a quantitative review", Archives of Physical Medicine and Rehabilitation, Vol. 77 No. 12, pp. 1226-1232.

Pallant, J. (2016), SPSS Survival Manual: A Step by Step Guide to Data Analysis Using IBM SPSS, 6th ed., Allen \& Unwin, Sydney, NSW.

Radomski, M.V. and Morrison, M.T. (2014), "Assessing abilities and capacities: cognition", in Radomski, M.V. and Latham, C.A.(Eds), Occupational Therapy for Physical Dysfunction, 7th ed., Wolters Kluwer Health/Lippincott Williams \& Wilkins, Philadelphia, PA, pp., 121-143.

Razani, J., Wong, J., Dafaeeboini, N., Edwards-Lee, T., Lu, P., Alessi, C. and Josephson, K. (2009), "Predicting everyday functional abilities of dementia patients with the Mini-Mental State Examination", Journal of Geriatric Psychiatry and Neurology, Vol. 22 No. 1, pp. 62-70.

Reppermund, S., Brodaty, H., Crawford, J.D., Kochan, N.A., Draper, B., Slavin, M.J., Trollor, J.N. and Sachdev, P.S. (2013), "Impairment in instrumental activities of daily living with high cognitive demand is an early marker of mild cognitive impairment: the Sydney memory and ageing study", Psychological Medicine, Vol. 43 No. 11, pp. 2437-2445.

Stineman, M.G., Shea, J.A., Jette, A., Tassoni, C.J., Ottenbacher, K.J., Fiedler, R. and Granger, C.V. (1996), "The Functional Independence Measure: tests of scaling assumptions, structure, and reliability across 20 diverse impairment categories", Archives of Physical Medicine and Rehabilitation, Vol. 77 No. 11, pp. 1101-1108.

Sweet, L., Van Adel, M., Metcalf, V., Wright, L., Harley, A., Leiva, R. and Taler, V. (2011), “The Montreal Cognitive Assessment (MoCA) in geriatric rehabilitation: psychometric properties and 
association with rehabilitation outcomes", International Psychogeriatrics, Vol. 23 No. 10, pp. 1582-1591.

Toglia, J., Fitzgerald, K.A., O’Dell, M.W., Mastrogiovanni, A.R. and Lin, C.D. (2011), “The Mini-Mental State Examination and Montreal Cognitive Assessment in persons with mild subacute stroke: relationship to functional outcome", Archives of Physical Medicine \& Rehabilitation, Vol. 92 No. 5, pp. 792-798.

Tombaugh, T.N. and McIntyre, N.J. (1992), “The Mini-Mental State Examination: a comprehensive review", Journal of the American Geriatrics Society, Vol. 40 No. 9, pp. 922-935.

Mini Mental State Examination

Tombaugh, T.N., McDowell, I., Kristjansson, B. and Hubley, A.M. (1996), "Mini-Mental State Examination (MMSE) and the modified MMSE (3Ms): a psychometric comparison and normative data", Psychological AssessmentVol. 8 No. 1, pp. 48.

Townsend, E. and Polatajko, H. (2013), Enabling Occupation II: Advancing an Occupational Therapy Vision for Health, Well-Being \& Justice through Occupation, 2nd ed., Canadian Association of Occupational Therapists Publications, Ottawa, ON.

University of Wollongong (2014), "What is the FIM ${ }^{\mathrm{TM}}$ instrument?", available at: http://ahsri.uow.edu. $\mathrm{au} /$ aroc/whatisfim/index.html (accessed 15 August 2015).

Wadley, V.G., Okonkwo, O., Crowe, M. and Ross-Meadows, L.A. (2008), "Mild cognitive impairment and everyday function: evidence of reduced speed in performing instrumental activities of daily living", American Journal of Geriatric Psychiatry, Vol. 16 No. 5, pp. 416-424.

Wales, K., Clemson, L., Lannin, N., Cameron, I. and Bayer, A. (2016), "Functional assessments used by occupational therapists with older adults at risk of activity and participation limitations: a systematic review", PLoS One, Vol. 11 No. 2, pp. e0147980.

Zheng, L., Teng, E.L., Varma, R., Mack, W.J., Mungas, D., Lu, P.H. and Chui, H.C. (2012), "Chineselanguage Montreal Cognitive Assessment for Cantonese or mandarin speakers: age, education, and gender effects", International Journal of Alzheimer's Disease, Vol. 2012 No. 204623.

Zwecker, M., Levenkrohn, S., Fleisig, Y., Zeilig, G., Ohry, A. and Adunsky, A. (2002), "Mini-Mental State Examination, cognitive FIM instrument, and the Loewenstein Occupational Therapy Cognitive Assessment: relation to functional outcome of stroke patients", Archives of Physical Medicine and Rehabilitation, Vol. 83 No. 3, pp. 342-345.

\section{Corresponding author}

Ted Brown can be contacted at: ted.brown@monash.edu

For instructions on how to order reprints of this article, please visit our website: 